\title{
Comment on the Letter to the editor: Bock et al. Occlusion of surgical opening of the ventricular system with fibrinogen-coated collagen fleece: a case collection study. Acta Neurochir (2011) 153:533-539
}

\author{
Hans Christoph Bock
}

Received: 12 November 2011 / Accepted: 15 November 2011 /Published online: 7 December 2011

(C) Springer-Verlag 2011

Thank you for your interesting comments outlining the sufficiency of collagen-fleece sealing for ventricular defects after carmustine wafer implantation. The use of fibrinogencoated collagen fleeces for sealing and reconstructive procedures of ventricular defects in neurosurgery has not yet been analysed by randomised clinical trials, but their use for neurosurgical indication as a local hemostatic agent with potent prevention of cerebrospinal fluid fistula has been reported in the literature, e.g. Nistor et al. (Skull Base Surg $7: 23-30,1997)$. We used the fleece for several complex hemostatic and dural-reconstructive indications, including cerebral as well as spinal operative procedures. The sponge-like consistency allows comfortable and accurate modelling, with a fast adhesive effect after direct fluid contact. We share the opinion of the authors that welldesigned studies are needed to analyse the potential benefit of using this agent for different neurosurgical indications compared with other biomaterials for reconstructive and watertight sealing strategies.

Conflicts of interest None.

H. C. Bock $(\bowtie)$

Department of Neurosurgery,

Johannes Gutenberg-University Mainz,

Langenbeckstraße 1,

55101 Mainz, Germany

e-mail: cbock@gmx.de 